\title{
David MURRAY, Poetry in Motion: Languages and Lyrics in the European Middle Ages
}

\section{Estelle Doudet}

\section{(2)enEdition}

\section{Journals}

Édition électronique

URL : https://journals.openedition.org/ccm/5092

DOI : $10.4000 / \mathrm{ccm} .5092$

ISSN : 2119-1026

\section{Éditeur}

Centre d'études supérieures de civilisation médiévale/Université de Poitiers

\section{Édition imprimée}

Date de publication : 1 septembre 2020

Pagination : 182-184

ISBN : 978-2-490783-06-9

ISSN : 0007-9731

Référence électronique

Estelle Doudet, "David murray, Poetry in Motion: Languages and Lyrics in the European Middle Ages », Cahiers de civilisation médiévale [En ligne], 250-251 | 2020, mis en ligne le 01 septembre 2020, consulté le 09 décembre 2022. URL : http://journals.openedition.org/ccm/5092 ; DOI : https://doi.org/10.4000/ ccm.5092

\section{(c) (i) (9)}

Creative Commons - Attribution - Pas d'Utilisation Commerciale - Pas de Modification 4.0 International - CC BY-NC-ND 4.0

https://creativecommons.org/licenses/by-nc-nd/4.0/ 
David Murray, Poetry in Motion: Languages and Lyrics in the European Middle Ages, Turnhout/Tours, Brepols/CESR (Épitome musical), 2019.

Le développement des histoires littéraires nationales au XIX ${ }^{\mathrm{e}} \mathrm{s}$. a eu pour conséquence de longtemps imposer une lecture réductrice des modes de production et de réception littéraires au Moyen Âge, en classant les auteurs, les œuvres, les récepteurs dans les bornes de langues et de pays définis par des critères modernes. Outre son anachronisme, ce type de classement rétrospectif a méconnu le fonctionnement des processus de création usuels aux $\mathrm{XII}^{\mathrm{e}}$ et XIII' $\mathrm{s}$. et a contribué à minimiser un aspect majeur des pratiques littéraires médiévales : la traduction-adaptation, le plurilinguisme, le code-switching, autrement dit la mouvance des langues jouant un rôle poétique majeur.

Réagissant à cette longue occultation, l'ouvrage de David Murray entend faire retour sur ces formes de créations translinguistiques en s'intéressant particulièrement à la poésie lyrique, art où elles ont été les plus florissantes et les plus significatives. Ce faisant, l'a. s'inscrit explicitement dans le sillage des recherches récentes activement menées en GrandeBretagne sur les langages poétiques médiévaux (le projet AHRC Medieval Francophone Literary Culture Outside France, dirigé par Simon GaunT, 2011-2015) ainsi que sur les savoirs véhiculés par la poésie au Moyen Âge (Une muse savante? Poésie et savoir, du Roman de la Rose jusqu'aux grands rhétoriqueurs, A. Armstrong et S. KAY [dir.], Paris, Classiques Garnier, 2014).

Trois raisons expliquent ce choix de corpus et d'approche. Pendant les $\mathrm{XII}^{\mathrm{e}}$ et $\mathrm{XIII}^{\mathrm{e}} \mathrm{s}$. que le livre prend pour périodes d'investigation, la composition poétique était indissociable de la performance lyrique. 
L'attention prêtée à la musique, au chant et à la voix en action a donné une acuité particulière aux questionnements sur les langues, matière de l'incantation poétique. De plus, à cette époque, plusieurs ensembles d'idiomes européens, tels que les langues germaniques et les langues romanes étudiées majoritairement par D. Murray, se présentaient sous la forme de continuums cohérents. Par ex., le français, l'occitan, le catalan ou le castillan étaient perçus par leurs locuteurs médiévaux comme des expressions certes différentes mais beaucoup plus interconnectées que des locuteurs d'aujourd'hui ne peuvent en avoir l'impression. Enfin, la poésie apparaissait elle-même comme un langage spécifique, caractérisé par des codes stylistiques, des modulations phonétiques et rythmiques, une syntaxe remodelée par la métrique. L'art lyrique pouvait donc être un laboratoire d'expériences et de questionnements sur les langages vernaculaires, leur potentiel créatif et leurs capacités de communication.

Telle est la thèse centrale que l'ouvrage de D. Murray s'attache à défendre et à illustrer. Elle n'est pas entièrement neuve, mais elle est soutenue par une démarche stimulante.

Le corpus sélectionné est un ensemble varié de poésies lyriques issues des régions occitanes, catalanes, galiciennes, castillanes, françaises, italiennes et germanophones. L'originalité est ici de traiter les poèmes et les langues à parts égales, en se laissant guider par le travail des poètes et les interprétations des récepteurs médiévaux. Le lecteur est amené à voyager de l'Espagne à la Sicile, d'Arras à Prague. Les poètes choisis présentent la même diversité : on lit des vers du troubadour Raimbaut d'Orange mais aussi d'Heinrich von Meissen dit Frauenlob, écrivain actifà la cour de Prague à la fin du XIII ${ }^{\mathrm{e}}$ s. Dans le même souci de variété, D. Murray a pris soin d'étudier des auteurs et des textes canoniques aussi bien que de mettre au jour des écrivains et des poèmes moins connus. La maîtrise avec laquelle $\mathrm{D}$. Murray circule à travers ces corpus pourrait sembler quelque peu étourdissante si son ouvrage n'était pas soigneusement articulé par des fils rouges bien visibles. Des poètes sont ainsi analysés à plusieurs reprises et de plusieurs points de vue au fil de l'étude : Bernard de Ventadour domine les chap. 1 et 2; Dante articule les chap. 2 et 3 ; l'œuvre de Cerveri, dernier troubadour catalan, est examinée aux chap. 2 et 4 . Le même souci de variété et d'organisation claire se fait jour dans le choix de grands textes théoriques sur les langages poétiques, du De vulgari eloquentia de Dante au Brevari de Matfré Ermengau, qui enrichissent l'analyse des corpus lyriques. L'ouvrage laisse ainsi transparaître un véritable effort d'équilibrage et de diversité des exemples, mis au service d'une démonstration ferme.

Le positionnement argumentatif de D. Murray est lui aussi solidement étayé. Il s'agit ici d'interroger les conceptions que les poètes européens des XII et $\mathrm{XIII}^{\mathrm{e}} \mathrm{s}$. ont développées sur le langage poétique en examinant les manières dont ils ont travaillé et fait travailler les langues vernaculaires de leur temps. Les quatre chapitres qui forment les points cardinaux de l'enquête illustrent par conséquent diverses formes de mobilité poétique et linguistique. L'ensemble dessine un itinéraire bien mené, allant de l'examen de poétiques singulières à l'étude de réceptions collectives et de leurs différents contextes socio-culturels.

En s'attachant à l'étude du phénomène bien connu qu'est la contrafacture (reprise de la structure métrique, rythmique ou musicale d'un poème dans une œuvre qui introduit un nouveau texte ou un nouveau sens), le chap. 1 met en œuvre une approche comparatiste consistant à analyser les réécritures translinguistiques et transhistoriques d'un chefd'œuvre, la canso Can vei la lauzeta composée par le troubadour Bernard de Ventadour au XII ${ }^{\mathrm{e}} \mathrm{s}$. L'approche comme l'ex. choisi sont déjà bien connus. Toutefois l'a. apporte un renouvellement intéressant en insistant sur les mutations des débats suscités par la célèbre chanson au fil de ses réécritures translinguistiques (débats sur les valeurs de l'amour, sur le style poétique). Il met par ailleurs en valeur la variété sociale des réceptions, le poème ayant circulé des cours courtoises vers le monde des clercs et des bourgeois.

Cette enquête introductive ayant permis de poser le vaste cadre chronologique et géographique qu'explore Poetry in Motion, le chap. 2 aborde le cas plus complexe de la poésie plurilingue. Il est approché à travers les deux phénomènes complémentaires de la Mehrsprachigkeit (le poème a une unité syntaxique dans une langue et intègre des vers en d'autres langues) et de la Sprachmischung (le poème développe son unité syntaxique et métrique en plusieurs langues). Du fameux poème Evas quan vey verdeyar, dont chaque strophe est composée dans une langue romane différente, à l'amusante tenso Domna tant vos ai preaida qui oppose le provençal Raimbaut de Vaqueiras à une génoise peu sensible à ses charmes, les exemples analysés ici jettent un éclairage particulièrement intéressant sur la théorisation du langage poétique comme lieu où convergent et se séparent symboliquement les langues romanes. Dans le poème plurilingue, celles-ci sont à la fois unies par le rythme et désunies par les incompréhensions qu'elles génèrent. 
Le troisième chapitre se penche dès lors sur les valeurs accordées aux langues dans la communication poétique. D'une manière convaincante, l'a. opère un élargissement du corpus étudié en y introduisant des œuvres réflexives : un traité, le De Vulgari Eloquentia de Dante; une encyclopédie, le Brevari de Matfré Ermengau; un roman, Le Roman de la rose ou de Guillaume de Dole de Jean Renart. Tous ces textes ont pour point commun d'intégrer dans leur tissu discursif des extraits de poèmes en d'autres langues et d'en faire l'impulsion d'une discussion sur les savoirs distillés par le chant lyrique : savoirs linguistiques, savoirs sentimentaux, savoirs culturels.

Élargissant ce questionnement, le chap. 4 démontre enfin les enjeux politiques des langages poétiques. Il s'appuie pour cela sur plusieurs régions et dynasties qui ont été soucieuses d'affirmer leur identité en promouvant la pratique lyrique de certaines langues, ainsi l'italien, langue littéraire encore minoritaire dans la Péninsule au XII ${ }^{\mathrm{e}}$ s., mais qui fut dominante en Sicile par la volonté du prince Hohenstaufen Frédéric II, ou des chansons allemandes du poète Frauenlob dont la diffusion en Bohème et en Europe centrale fut soutenue par la dynastie germanophone des Premyslides.

Fermement argumenté, remarquablement détaillé dans ses analyses, fort d'un corpus d'une rare richesse, Poetry in Motion est assurément appelé à devenir un livre important parmi les études sur la poésie et la culture linguistique médiévales. Son ambition méthodologique est particulièrement remarquable, quoiqu'elle appelle deux brèves remarques. Un petit regret est causé par l'absence presque complète d'analyse mélodique (et d'insertions de portées, sauf p. 119-121), ce qui ne laisse pas de surprendre pour un livre publié dans la collection «Épitome musical». Or, on l'a dit, l'un des points forts de Poetry in Motion est bien de rendre toute sa place à la dimension lyrique de la poésie médiévale, un élément essentiel des réflexions des poètes sur le langage poétique. Le manque d'étude de la musique se fait donc sentir, même si cette étude est annoncée comme un «challenge » (p. 250) que l'a. devrait relever dans ses prochaines publications. Par ailleurs, le choix d'une démarche privilégiant la mobilité est incontestablement un apport important de l'ouvrage de D. Murray. Poetry in Motion illustre avec brio le renouvellement de perspective que revendiquent aujourd'hui les Mobility Studies bien développées outre-Atlantique aussi bien que l'histoire connectée des productions culturelles qu'expérimentent les historiennes et historiens francophones. On est dès lors étonné de voir D. Murray, dans une conclusion au demeurant trop brève (p. 247-251), expliquer sa démarche en revendiquant le seul héritage d'ErnstRobert Curtius. Certes, le terrain d'enquête chronologique et linguistique de Poetry in Motion est proche de celui d'Europäische Literatur und lateinisches Mittelalter, l'ouvrage pionnier du savant allemand (Ernst-Robert CURTIUS, La littérature européenne et le Moyen Âge latin, J. BRÉJoux [trad.], Paris, Presses universitaires de France, 1956, [1 $1^{\text {re }}$ éd. all. 1948]). Toutefois, il semble que les objectifs soient sensiblement différents : la Toposforschung de E.-R. Curtius suppose que le topos reste stable au fil de ses réutilisations successives, alors que l'enquête de D. Murray insiste sur les inflexions de sens et de rythme suscitées par la circulation d'un chant lyrique à travers plusieurs langues. En revanche, le traitement des corpus à l'écart de tout récit centralisateur offre ici un certain écho à «l'histoire à parts égales » théorisée par Romain Bertrand (Romain BERTRAND, L'Histoire à parts égales, récits d'une rencontre Orient-Occident [XVI ${ }^{e}-X V I I^{e}$ s.], Paris Seuil, 2011). De même, l'attention minutieuse que l'a. porte aux opérations de transfert poétique et linguistique fait écho aux pratiques de l'histoire culturelle connectée, l'une des approches impulsées par le global turn des méthodes historiques (Michel EsPagNe, « La notion de transfert culturel », Revue Sciences/Lettres, 1, 2013, DOI : 10.4000/rsl.219). Cette convergence est d'ailleurs sensible dès le premier chapitre de Poetry in Motion, qui questionne la démarche comparatiste en des termes assez voisins de ceux de l'histoire connectée. On regrette donc que l'a., fort d'une approche fermement maîtrisée, n'ait pas plus clairement précisé son positionnement épistémologique dans les dernières pages de son livre. Il s'agit toutefois de points que les futures publications de D. Murray pourront préciser. Reste un ouvrage fermement écrit, solidement pensé et riche d'exemples passionnants qui ne manqueront pas d'intéresser les historiens des langues, des littératures et de la sociologie des pratiques culturelles médiévales.

Estelle DoudeT

UMR 5316 - LITT\&ARTS

Université de Grenoble Alpes/ Université de Lausanne 